\title{
Methods for controlling of the laser-induced absorption in a BTO crystal by using of cw-laser radiation
}

\author{
A. Matusevich • A. Tolstik • M. Kisteneva • \\ S. Shandarov • V. Matusevich • A. Kiessling • \\ R. Kowarschik
}

Received: 2 November 2008 / Revised version: 19 March 2009 / Published online: 19 April 2009

(C) Springer-Verlag 2009

\begin{abstract}
A photorefractive BTO crystal is exposed with cw-laser beams $\left(430 \mathrm{~mW} / \mathrm{cm}^{2}\right.$ at $\left.514 \mathrm{~nm}\right)$, and the photoinduced absorption between 480 and $900 \mathrm{~nm}$ is studied. A method of controlling this absorption by low intensity laser radiation of "green" and "red" wavelengths is demonstrated. The physical mechanism can be explained by the redistribution of electrons on the long-lived energy levels into the forbidden band. Dynamical characteristics of the redistribution are estimated.
\end{abstract}

PACS 42.70.Mp $\cdot 42.70 . \mathrm{Gi}$

\section{Introduction}

The photo-induced absorption is the change of the absorption of light as the result of irradiation. Any influence of light on the internal structure of the photorefractive crystals, which is connected with the energy redistribution of charge carriers, can change the absorption. The temporal and amplitude behavior of this effect depend strongly on the energy levels and the relaxation characteristics of the materials. Most of the characteristic effects are often disregarded in

A. Matusevich $(\varangle) \cdot$ V. Matusevich · A. Kiessling .

R. Kowarschik

Institute of Applied Optics, Friedrich-Schiller-University,

07743 Jena, Germany

e-mail: Andrew.Matusevich@uni-jena.de

Fax: +49-(0)3641-947652

M. Kisteneva $\cdot$ S. Shandarov

Department of Electronic Devices, State University of Control

Systems and Radioelectronics, Tomsk, Russia

A. Tolstik

Belarusian State University, Minsk, Belarus the quasi-stationary processes because of their short lifetime 1-100 ps or weak exposure intensities [1]. Sometimes the induced absorption can have life-times in the order of seconds, minutes, hours, and even days.

All crystals of the sillenite group $\mathrm{Bi}_{12} \mathrm{MO}_{20}$ (where $\mathrm{M}=$ $\mathrm{Si}, \mathrm{Ti}, \mathrm{Ge}$ ) show long-lived induced absorption under external irradiation. This induced response is caused by the redistribution of charge carriers on the traps in the valence band. The structure of the traps depends on impurities. This way impurities have strong influence on the spectrum of the photo-induced absorption, which was shown by Martin et al. and Briat et al. for $\mathrm{Bi}_{12} \mathrm{SiO}_{20}$ and $\mathrm{Bi}_{12} \mathrm{GeO}_{20}$ [1-9] since 1991. Moreover, Martin et al. showed that the induced absorption in the pure crystal can be much less than the absorption of doped sillenite crystals and in some cases could be neglected. Martin et al. and Panchenko analyzed also the dependence of the absorption on the temperature for doped and undoped crystals [1-6, 10]. In 1999, Kobozev et al. investigated the light-induced absorption in $\mathrm{Bi}_{12} \mathrm{TiO}_{20}$ (BTO) [11] and tried to describe the effect by three-valence-states impurity-centers in combination with shallow traps. In 2003, Marinova et al. studied light-induced properties of $\mathrm{Bi}_{12} \mathrm{TiO}_{20}: \mathrm{Ru}$ and showed the influence of impurity concentrations on absorption and photo-induced absorption [12]. In 2002, Shandarov et al. [13] investigated experimentally the photo-induced absorption in $\mathrm{Bi}_{12} \mathrm{TiO}_{20}: \mathrm{Ca}$ and suggested a three-level model, which is a special case of the two-center model. The lifetime of the induced absorption was about several hours and depended on the temperature [14]. In 2005, they reported the photo-induced absorption in planar waveguides based on $\mathrm{Bi}_{12} \mathrm{TiO}_{20} / \mathrm{Bi}_{12} \mathrm{SiO}_{20}$ and $\mathrm{Bi}_{12} \mathrm{TiO}_{20}: \mathrm{Cu} / \mathrm{Bi}_{12} \mathrm{SiO}_{20}$ crystals [15]. In 2007, we investigated the spectral characteristics of the photo-induced absorption in the pure $\mathrm{Bi}_{12} \mathrm{TiO}_{20}$ using pulse lasers and calculated the energy of the absorption centers [16]. It should 
be noticed that photo-induced absorption in $\mathrm{Bi}_{12} \mathrm{TiO}_{20}$ crystals was experimentally found already in the past and was described as the reason for the absorption grating [17, 18].

Recently we have presented investigations of the longlived absorption that was photo-induced by high intensity pulsed-laser beams $\left(2 \mathrm{MW} / \mathrm{cm}^{2}\right.$ pro $40 \mathrm{~ns}$ at $\left.532 \mathrm{~nm}\right)$ in photorefractive $\mathrm{Bi}_{12} \mathrm{TiO}_{20}$ crystals. We noticed that the absorption increased further up to $900 \mathrm{~nm}$ and explained this effect by the existence of long-lived levels in the forbidden band where the charges are collected after quick relaxation from the conducting band. Analyzing the relaxation times for different wavelengths, we found that it is possible to use the two-center model. There are primary two longlived centers (levels) with lifetimes of ca. $10^{4}$ and $10^{5} \mathrm{~s}$ which we have experimentally found. The photo-induced absorption has a hysteresis character which can be seen in the $k_{600}-k_{800}$ diagram ( $k_{600}$ and $k_{800}$ are the corresponding absorption coefficients at the wavelengths 600 and $800 \mathrm{~nm}$, respectively) of the development of the absorption during excitation-relaxation processes [19].

The present paper is the continuation of our investigation of the long-lived photo induced absorption in a $\mathrm{Bi}_{12} \mathrm{TiO}_{20}$ crystal with a lifetime in the order of minutes. For a better understanding of the temporal behavior of the internal processes in the crystal, we are now using cw-laser radiation. We show experimental methods which give the opportunity to influence the behavior of induced absorption. These methods give the possibility to decrease the induced effect or, on the other hand, to stabilize it in $\mathrm{Bi}_{12} \mathrm{TiO}_{20}$ crystals. A qualitative explanation of the underlying processes is given. The experimental dynamical characteristics and the recombination coefficients are estimated.
2 Photo-induced absorption in a BTO crystal generated by a cw-laser

The investigation of the photo-induced absorption in BTO was carried out for a nominally pure $\mathrm{Bi}_{12} \mathrm{TiO}_{20}$ which was grown from a congruent melt by the Czochralski method. It has the cubic symmetry 23 , like all representatives of the sillenite family. The light radiation propagated along the [100] crystallographic axis. The (100) faces with transverse dimensions of $7 \times 7 \mathrm{~mm}^{2}$ were polished to optical quality. The thickness of the crystal in the [100] direction was $2.8 \mathrm{~mm}$. The light induced absorption was realized by a cw-laser at $514 \mathrm{~nm}\left(\mathrm{Ar}^{+}\right)$(Fig. 1(a)). Low intensity lasers at $532 \mathrm{~nm}$ (Nd:YAG) and $633 \mathrm{~nm}(\mathrm{He}-\mathrm{Ne})$ with intensities up to 12 and $17 \mathrm{~mW} / \mathrm{cm}^{2}$, respectively, were additionally used for controlling the photo-induced absorption. The average intensity of the laser radiation incident on the crystal during the exposition was $430 \mathrm{~mW} / \mathrm{cm}^{2}$. The polarization of the laser light was located in the plane (100) and parallel to [011]. The absorption of the media was measured with a spectrophotometer based on a CCD line array with 2048 pixels.

\subsection{Excitation of the photo-induced absorption in a BTO crystal by a cw-laser}

In Figs. 2(a) and 2(b), the spectra of common and photoinduced absorption against the exposition energy are shown. A cw-laser with a wavelength of $514 \mathrm{~nm}$ was used for inducing of absorption.

The effect of the photo-induced absorption can be explained by long-lived centers with the energy levels in the
Fig. 1 A scheme of the experimental setup for the generation and measuring of the photo-induced absorption. Laser $514 \mathrm{~nm}-\mathrm{Ar}^{+}(514 \mathrm{~nm})$ cw-laser, Laser $532 \mathrm{~nm}-\mathrm{Nd}: Y A G(532 \mathrm{~nm})$ cw-laser, Laser $633 \mathrm{~nm}-\mathrm{He}-\mathrm{Ne}(633 \mathrm{~nm})$ cw-laser, TS-telescopic system, D-diaphragm, BS—beam splitter, M-mirror, DM-dichroitic mirror AR@532 nm/HR@633 nm, $\mathrm{Xe} / \mathrm{HL}-\mathrm{Xe} / \mathrm{halogen}$ lamp (200-1000 nm), F-fiber optics, $\mathrm{C}$-collimator,

S-spectrophotometer, $\mathrm{CM}$-collimating mirror, DG-diffraction grating, CCD — CCD linear array "Sony 2048", Det-photo detector, PC-computer

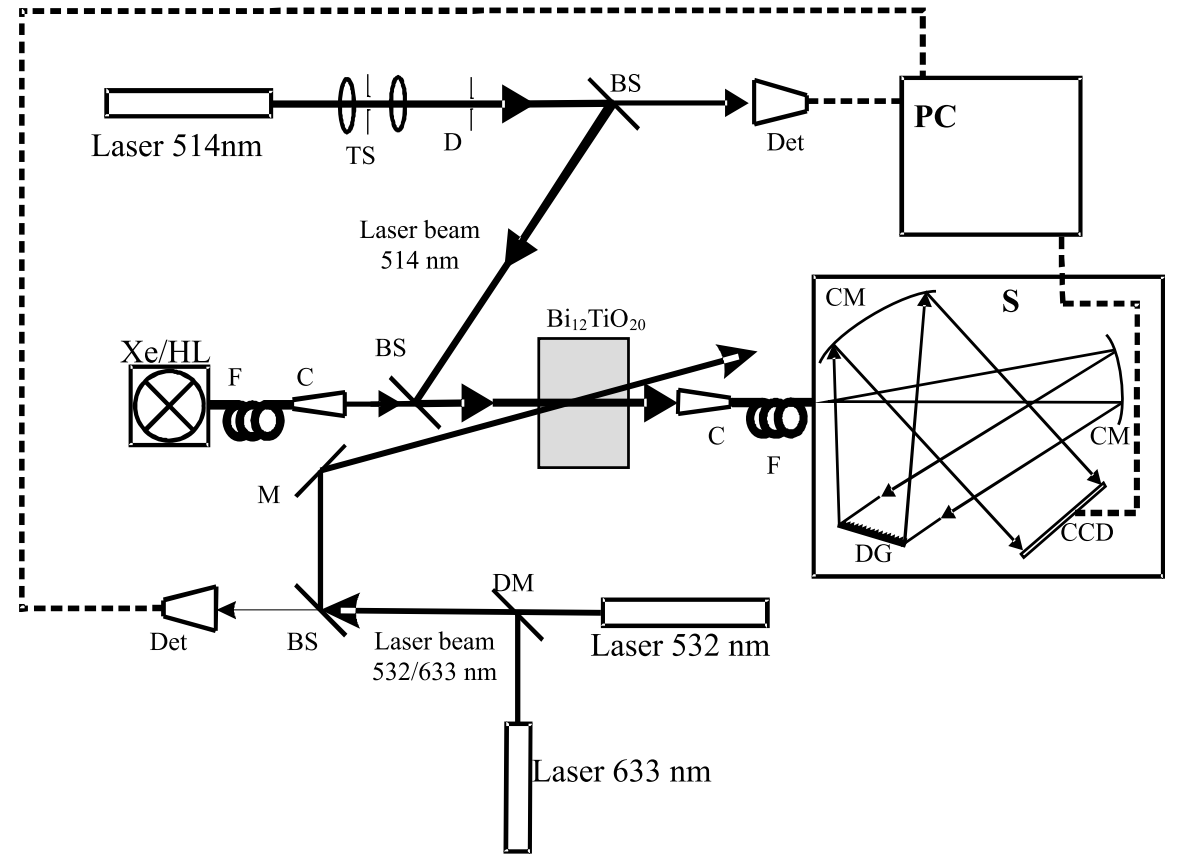




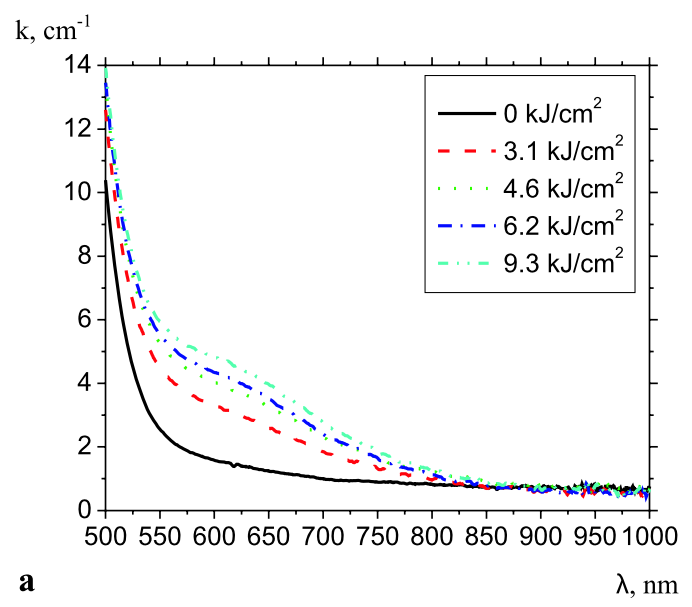

$\mathrm{k}_{\mathrm{PI}}, \mathrm{cm}^{-1}$

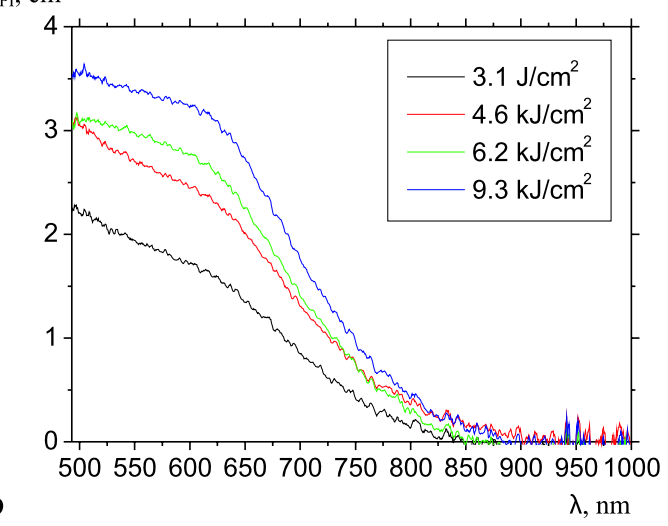

Fig. 2 (a) Absorption spectrum of BTO for different values of the total exposition $\left(\mathrm{J} / \mathrm{cm}^{2}\right) ; k$ is the absorption coefficient. (b) Dependence of the photo-induced absorption $k_{\mathrm{PI}}$ (difference between integral absorption $k$ and initial absorption without exposition $k_{0}$ ) for $\mathrm{cw}$-laser $\left(430 \mathrm{~mW} / \mathrm{cm}^{2}\right.$ at $\left.514 \mathrm{~nm}\right)$

forbidden band that are caused by impurities and defects in the bulk of the crystal [16]. These centers can be denoted as intermediate-level traps (IT) $[2,3,19]$. It was shown by Oberschmid [20] for bismuth oxides of the type $\mathrm{Bi}_{12} \mathrm{MO}_{20}$ (where $\mathrm{M}=\mathrm{Ge}, \mathrm{Si}$ or $\mathrm{Ti}$ ) that the incorrect occupation by $\mathrm{Bi}_{\mathrm{M}}$ is responsible for the light absorption in the region between 2.3 and $3.2 \mathrm{eV}$ and causes the deep traps (DT). Investigations of Reyher et al. and Vogt et al. [21, 22] show that the nature of the IT is determined by a $\mathrm{Bi}^{3+}$ ion associated with a hole, whereas the hole is mainly localized at the oxygen neighbors. As it was shown in [19] for an irradiation at 514 and $532 \mathrm{~nm}$, the excitation of electrons basically takes place from DT to the conducting band (CB). If the charges are excited into the $\mathrm{CB}$ they can recombine back to the valence band (VB) and DT as well as to the long-lived IT. The charges of the long-lived levels can be excited again into the CB by photons with lower energy. A growth of the photo-induced absorption $k_{\mathrm{PI}}$ is observed at wavelengths below $900 \mathrm{~nm}$ (Fig. 2(b)). The photo-induced absorption $k_{\mathrm{PI}}$ is $\mathrm{k}, \mathrm{cm}^{-1}$

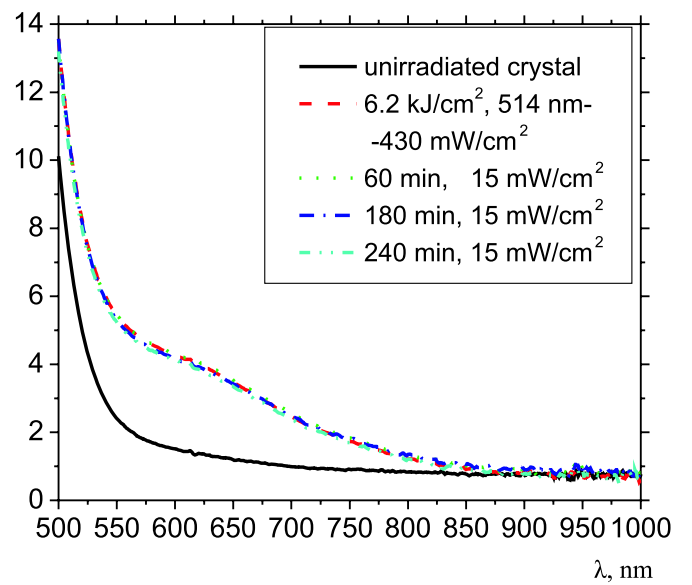

Fig. 3 Influence of the cw-radiation with the low intensity $15 \mathrm{~mW} / \mathrm{cm}^{2}$ and wavelength $514 \mathrm{~nm}$ on changing of the photo-induced absorption of BTO. The absorption is generated by cw-radiation $\left(430 \mathrm{~mW} / \mathrm{cm}^{2}\right.$ at $\left.514 \mathrm{~nm}\right)$

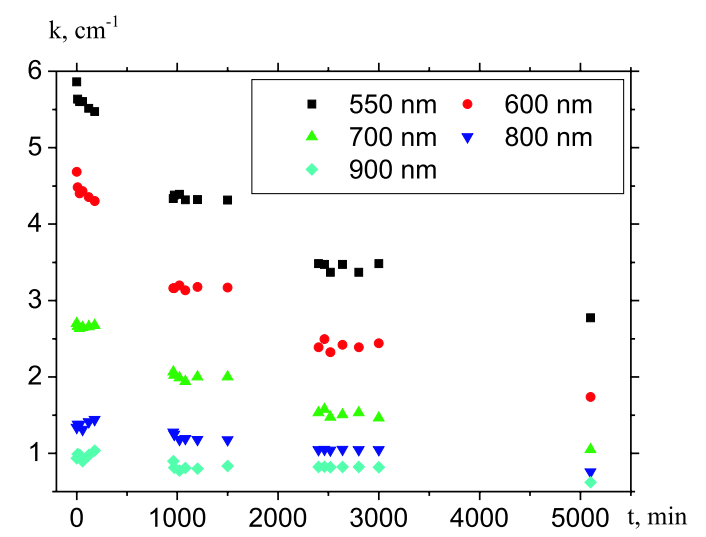

Fig. 4 An example for the temporal retardation of the relaxation at different stages by a cw-radiation with low intensity $12 \mathrm{~mW} / \mathrm{cm}^{2}$ at $532 \mathrm{~nm}$ in BTO. It demonstrates the dependence of the coefficient of absorption on the time of free relaxation and cw-radiation at $532 \mathrm{~nm}$ for the given wavelengths; during the free relaxation, the $\mathrm{cw}$-radiation at $532 \mathrm{~nm}$ is switched on for 0-180 min., 970-1500 min., 2400-3000 min (there are three plateaus at the curves)

connected with the excitation of charges from the IT to the CB.

\subsection{Control of the photo-induced absorption in a BTO} crystal by low intensity cw-lasers

The relaxation of the photo-induced absorption can be slowed down using cw-radiation with low intensity (Figs. 3, 4). If the photo-induced absorption is generated with an intensity of $430 \mathrm{~mW} / \mathrm{cm}^{2}$ at $514 \mathrm{~nm}$, it is enough to use $\mathrm{cw}$-radiation at the wavelengths 532 and $514 \mathrm{~nm}$ with low intensities (e.g., 12 and $15 \mathrm{~mW} / \mathrm{cm}^{2}$, respectively) to stop the relaxation process (Fig. 3). In such a way, it is possi- 


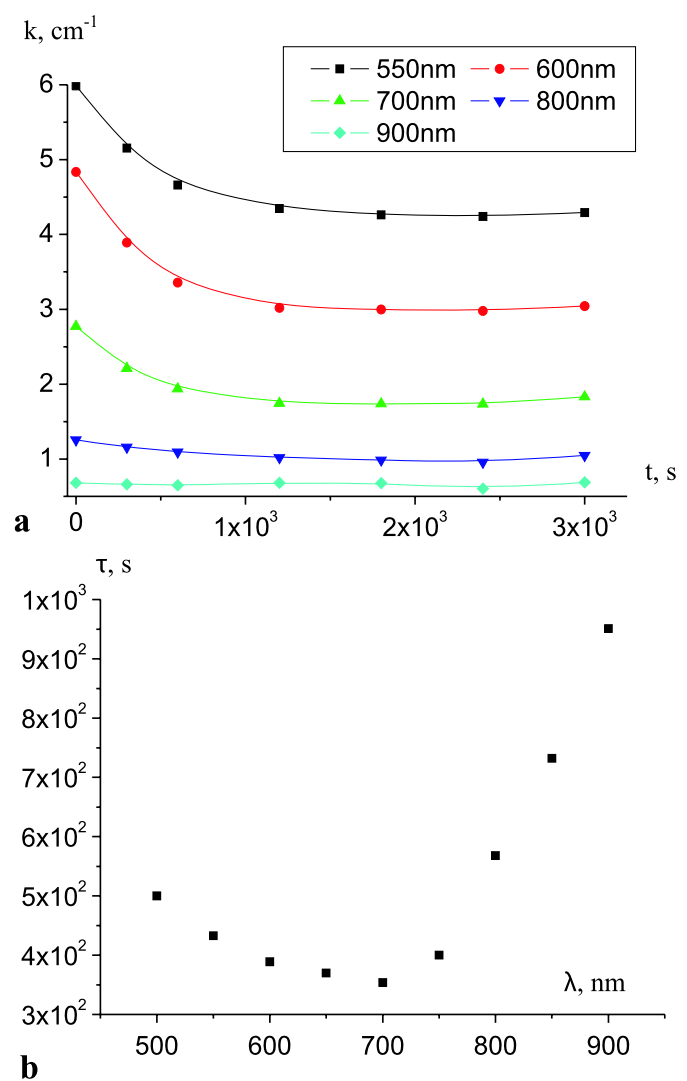

Fig. 5 An example for the temporal acceleration of the relaxation at different stages by a cw-radiation with low intensity $17 \mathrm{~mW} / \mathrm{cm}^{2}$ at $633 \mathrm{~nm}$ in BTO. (a) Dependence of the coefficient of absorption for the given wavelengths on the time of exposition, (b) dependence of the calculated long-life centers lifetimes on the probe beam wavelength

ble to stop the relaxation at any stage. If one switches off the low cw-radiation, the relaxation process resumes (Fig. 4).

If the BTO crystal with the recently photo-induced absorption is exposed by a cw-radiation at $633 \mathrm{~nm}$ with low intensity (e.g., $17 \mathrm{~mW} / \mathrm{cm}^{2}$ ), the relaxation is accelerated. If one switches off the low cw-radiation of $633 \mathrm{~nm}$, the relaxation process resumes with the evolution as without previous acceleration (Fig. 5). One can calculate the exponential lifetime of the photo-induced absorption under stimulation of the relaxation (Fig. 5(b)). Compared with the results from [19] for the unstimulated relaxation red light accelerates the relaxation approximately by a factor in the range of 10-100.

Low intensity cw-radiation of the wavelengths 514,532 , and $633 \mathrm{~nm}$ did not produce enough photo-induced absorption to be experimentally detected. Thus, one can use low intensity expositions at different wavelengths in order to control the photo-induced absorption.

Qualitatively, the mechanism of induced absorption can be explained by the scheme in Fig. 6. The long-lived absorption is connected with a population of the IT. During relaxation of the crystal the number of absorption centers at the trap levels decreases with approximate lifetimes of $10^{4}$

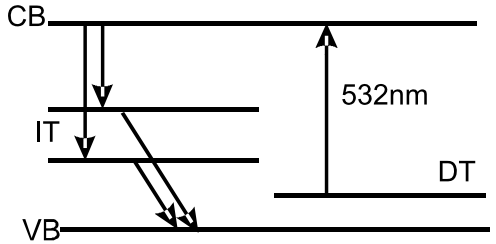

a

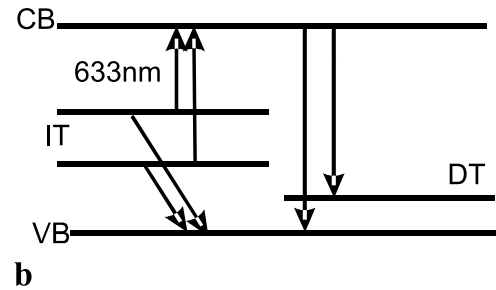

Fig. 6 Schematic illustration of the photo-induced absorption in BTO controlled by "green" and "red" radiations: (a) stopping of the relaxation by light at $532 \mathrm{~nm}$, (b) speeding of the relaxation by light at $633 \mathrm{~nm}$

and $10^{5} \mathrm{~s}$ [19]. This quantity can be kept invariable. Using low intensity exposition of "green" light, one transfers electrons from DT to IT. When the number of transferred electrons is equal to the number of relaxed ones from IT to VB or DT, then the absorption remains constant (Fig. 6(a)). The induced absorption relaxation can be accelerated by low intensity "red" radiation. In this case, the radiation excites electrons from IT to $\mathrm{CB}$ with a following relaxation to the VB. Together with the normal relaxation this speeds up the decreasing of the absorption (Fig. 6(b)).

According to the experimental results, one is able to write down the coupling equation for describing of the BTO dynamical characteristics, which is also schematically illustrated in Fig. 7:

$$
\begin{aligned}
\frac{\partial N_{1}}{\partial t}= & -S_{1} I N_{1}+r_{3,1} N_{3} N_{1}^{+}+r_{2 a, 1} N_{1}^{+} N_{2 a} \\
& +r_{2 b, 1} N_{1}^{+} N_{2 b}, \\
\frac{\partial N_{2 a}}{\partial t}= & -S_{2 a} I N_{2 a}+r_{3,2 a} N_{3} N_{2 a}^{+}-r_{2 a, 1} N_{1}^{+} N_{2 a} \\
& -\beta_{a, b} N_{2 a}+\beta_{b, a} N_{2 b}, \\
\frac{\partial N_{2 b}}{\partial t}= & -S_{2 b} I N_{2 b}+r_{3,2 b} N_{3} N_{2 b}^{+}-r_{2 b, 1} N_{1}^{+} N_{2 b} \\
& +\beta_{a, b} N_{2 a}-\beta_{b, a} N_{2 b}, \\
\frac{\partial N_{3}}{\partial t}= & S_{1} I N_{1}+S_{2 a} I N_{2 a}+S_{2 b} I N_{2 b}-r_{3,1} N_{3} N_{1}^{+} \\
& -r_{3,2 a} N_{3} N_{2 a}^{+}-r_{3,2 b} N_{3} N_{2 b}^{+} .
\end{aligned}
$$

$N_{1}, N_{2 a}, N_{2 b}, N_{3}$ are the concentrations of the charge carries (electrons) involved in the phenomena at the levels of deeps traps, lower long-lived traps, upper long-lived traps and the conducting band, respectively. $N_{1}^{+}, N_{2 a}^{+}, N_{2 b}^{+}$ 
Fig. 7 Schematic illustration of electron transitions between levels of the photo-induced absorption in BTO

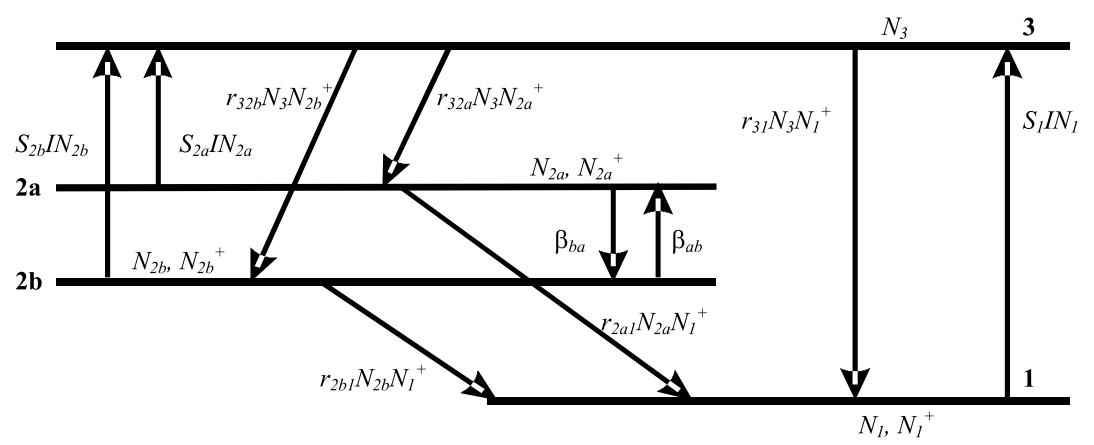

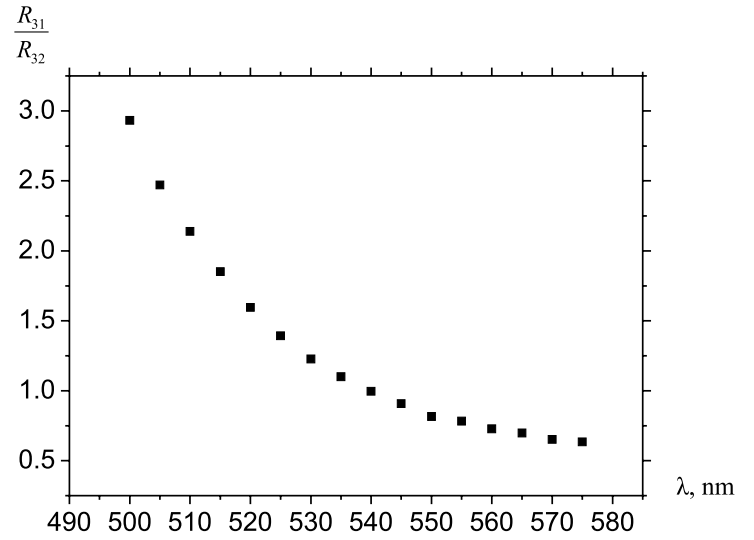

Fig. 8 Dependence of the ratio of the effective recombination coefficients on the stimulation wavelength

are the corresponding concentrations of the ionized centers (ready to "receive" an electron) at the levels of the traps. $S_{1}, S_{2 a}, S_{2 b}$ are cross-sections of the light quantum absorption during excitation of the electrons from traps to CB. $S_{i}$ is a function of the light frequency $S_{i}(\omega)$ and the depth of the level in the forbidden band. $r_{i, j}$ are the recombination coefficients from the upper level $i$ to the lower $j . \beta_{a, b}$ and $\beta_{b, a}$ are the constants defining inner transitions coefficients between long-lived levels caused by thermal excitation, recombination, tunneling and etc. According to the initial spectrum of the absorption and especially low absorption in the longwave range, one may ignore thermal ionization between DT, IT and CB. Low long-wave absorption at the final spectrum means insignificant light induced transition of the electrons between the long-lived traps. $I$ is the intensity of the homogenous and uniform light with the frequency $\omega$ measured in photon $/ \mathrm{m}^{2} \mathrm{~s}$.

As a result of the initial electric neutrality of the crystal we get

$N_{1}+N_{2 a}+N_{2 b}+N_{3}=N_{1}^{+}+N_{2 a}^{+}+N_{2 b}^{+}+N_{3}^{+}$.

In such a way, the absorption coefficients of the light for the wavelengths 514 and $532 \mathrm{~nm}$ have the form:

$k=S_{1} N_{1}+S_{2 a} N_{2 a}+S_{2 b} N_{2 b}$.

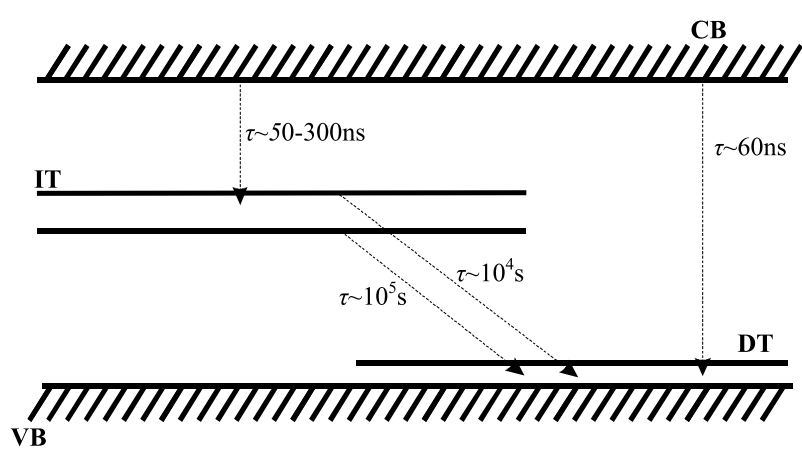

Fig. 9 Dynamical characteristics of the nominally pure $\mathrm{Bi}_{12} \mathrm{TiO}_{20}$

Table 1 Dynamical characteristics of the nominally pure $\mathrm{Bi}_{12} \mathrm{TiO}_{20}$

\begin{tabular}{lll}
\hline$\lambda, \mathrm{nm}$ & 514 & 532 \\
\hline$\tau_{2 a, 1}, \mathrm{~s}[19]$ & $1.2 \times 10^{4}$ & $1 \times 10^{4}$ \\
$\tau_{2 b, 1} \mathrm{~s}[19]$ & $5.5 \times 10^{4}$ & $7.5 \times 10^{4}$ \\
$\tau_{3,1}=\frac{1}{R_{3,1}}, \mathrm{~s}[23]$ & $\sim 6 \times 10^{-8}$ & $\sim 6 \times 10^{-8}$ \\
$\tau_{3,2}=\frac{1}{R_{3,2}}, \mathrm{~s}$ & $\sim 12 \times 10^{-8}$ & $\sim 7 \times 10^{-8}$ \\
\hline
\end{tabular}

The corresponding photo-induced absorption is:

$k_{p i}=S_{2 a} N_{2 a}+S_{2 b} N_{2 b}$.

We now introduce the following parameters:

1. $r_{2,1}$ is the average recombination coefficient:

$r_{2,1}=\frac{r_{2 a, 1} \cdot N_{2 a}+r_{2 b, 1} \cdot N_{2 b}}{N_{2 a}+N_{2 b}}$.

Physically it means a common probability of recombination of the electrons from IT to DT.

2. $r_{3,2}$ is the average recombination coefficient for recombination of the electrons from CB to IT:

$r_{3,2}=\frac{r_{3,2 a} \cdot N_{2 a}^{+}+r_{3,2 b} \cdot N_{2 b}^{+}}{N_{2 a}^{+}+N_{2 b}^{+}}$ 
$S_{2}$ is the average absorptions cross section for the IT:

$$
S_{2}=\frac{S_{2 a} \cdot N_{2 a}+S_{2 b} \cdot N_{2 b}}{N_{2 a}+N_{2 b}} .
$$

After the summation of the (2) and (3) and considering (8)(10) we can write (1)-(4) as follows:

$$
\begin{gathered}
\frac{\partial N_{1}}{\partial t}=-S_{1} I N_{1}+r_{3,1} N_{3} N_{1}^{+}+r_{2,1} N_{1}^{+}\left(N_{2 a}+N_{2 b}\right) \\
\frac{\partial\left(N_{2 a}+N_{2 b}\right)}{\partial t}=-S_{2} I\left(N_{2 a}+N_{2 b}\right)+r_{3,2} N_{3}\left(N_{2 a}^{+}+N_{2 b}^{+}\right) \\
-r_{2,1} N_{1}^{+}\left(N_{2 a}+N_{2 b}\right),
\end{gathered}
$$

$$
\begin{aligned}
\frac{\partial N_{3}}{\partial t}= & S_{1} I N_{1}+S_{2} I\left(N_{2 a}+N_{2 b}\right)-r_{3,1} N_{3} N_{1}^{+} \\
& -r_{3,2} N_{3}\left(N_{2 a}^{+}+N_{2 b}^{+}\right) .
\end{aligned}
$$

A new parameter $N_{2}$ can be introduced now:

$N_{2}=N_{2 a}+N_{2 b}$

Physically $N_{2}$ describes the total concentration of charge carriers located at the IT levels.

As it was shown in the experiment (Fig. 3), when one used a low intensity radiation from the wave range response for inducing of the long-lived absorption, relaxation stopped. This means that the system was in equilibrium.

Under this conditions (Fig. 3) the adiabatic approximation $\frac{\partial N_{i}}{\partial t}=0$ can be used, and (11)-(13) take the from:

$0=-S_{1} I N_{1}+r_{3,1} N_{3} N_{1}^{+}+r_{2,1} N_{1}^{+} N_{2}$,

$0=-S_{2} I N_{2}+r_{3,2} N_{3} N_{2}^{+}-r_{2,1} N_{1}^{+} N_{2}$,

$0=S_{1} I N_{1}+S_{2} I N_{2}-r_{3,1} N_{3} N_{1}^{+}-r_{3,2} N_{3} N_{2}^{+}$.

The characteristic recombination time of the electrons from the CB to the DT and VB is of the order of $10 \mathrm{~ns}$ [23]. On the other hand, the characteristic recombination time of the electrons from the IT to the DT and VB averages out to hours [19]. That is why:

$r_{3,1} \gg r_{2,1}$

and (15) takes the form:

$S_{1} I N_{1}=r_{3,1} N_{3} N_{1}^{+}$.

Taking into account (17) and (19), it yields:

$$
S_{2} I N_{2}=r_{3,2} N_{3} N_{2}^{+}
$$

and, from the correlation of (19) and (20), we get:

$$
\frac{S_{1} N_{1}}{S_{2} N_{2}}=\frac{r_{3,1} N_{1}^{+}}{r_{3,2} N_{2}^{+}} .
$$

In other words,

$\frac{k_{0}}{k_{\mathrm{PI}}}=\frac{R_{3,1}}{R_{3,2}}$,

where $k_{0}$ and $k_{\mathrm{PI}}$ are the initial and the photo-induced absorption coefficients reached during the "relaxation stopping" experiment. $R_{3,1}$ and $R_{3,2}$ are the relaxation constants from CB to DT and IT, respectively. $R_{3,1}$ and $R_{3,2}$ are inverse parameters to the lifetimes of the electrons at the levels $\tau_{3,1}$ and $\tau_{3,2}$. So, one finds the ratio between the electron recombination from $\mathrm{CB}$ to long-lived IT and DT. This ratio shows that the electrons ionized to the $\mathrm{CB}$ have a relaxation probability to the long-lived IT, which is 1.2 times lower than that to the DT for $532 \mathrm{~nm}$ (1.9 for $514 \mathrm{~nm}$ ).

Our conclusions may be disseminated to all the wavelengths of the range, which can induce the absorption. For wavelengths of the stimulation range one can present a dependence of the ratio (22) on the wavelength (Fig. 8). According to the spectrum of the initial absorption (Fig. 2(a)), the stimulation range is to the left of the $580 \mathrm{~nm}$. However, this border is changing and depends on the complex nature of the traps.

The dependence on the wavelength is the result of the broad long-lived energy level and of the various concentrations of the absorption centers therein.

According to this result and to our previous experimental investigations [19, 23], one can estimate the dynamical characteristics of the levels (traps) (Table 1) illustrated in Fig. 9.

\section{Conclusion}

In conclusion, we exposed the BTO crystal with cw-laser beams $(430 \mathrm{~mW}$ at $514 \mathrm{~nm})$ and investigated the photoinduced absorption. We found that the absorption increased further in a range up to $850 \mathrm{~nm}$, and we explained this effect by the existence of long-lived levels in the forbidden band where the charges are collected after quick relaxation from the conducting band. A method for controlling this absorption by low intensity laser radiation of "green" and "red" wavelengths was demonstrated. The "green" low intensity exposition (e.g., 12 and $15 \mathrm{~mW} / \mathrm{cm}^{2}$ at the wavelengths 532 and $514 \mathrm{~nm}$, respectively) can retard the relaxation process, while the "red" low intensity exposition (e.g., $17 \mathrm{~mW} / \mathrm{cm}^{2}$ at $633 \mathrm{~nm}$ ) can accelerate it by a factor of ten. However, low intensity cw-radiation does not induce absorption. The internal dynamic characteristics of band-to-band recombination were estimated on the base of the experimental results. For the crystal $\mathrm{Bi}_{12} \mathrm{TiO}_{20}$ they are: $\tau \sim 60 \mathrm{~ns}$ for $\mathrm{CB}-\mathrm{VB}$ recombination, $\tau \sim 50-300$ ns for $\mathrm{CB}-\mathrm{IT}$ recombination, $\tau \sim 10^{5}, 10^{4} \mathrm{~s}$ for IT-VB/DT recombination. 


\section{References}

1. J. J Martin, I. Foldvari, C.A. Hunt, J. Appl. Phys. 70, 7554 (1991)

2. D.W. Hart, C.A. Hunt, D.D. Hunt, J. J Martin, M.T. Harris, J.J. Larkin, J. Appl. Phys. 73, 1443 (1993)

3. J.S. McCullough, A. Harmon, J. J Martin, M.T. Harris, J.J. Larkin, J. Appl. Phys. 78, 2010 (1995)

4. J.S. McCullough, A.M. Georgalas, C.A. Hunt, S.P. HoeflerCoster, D.W. Peakheart, G.S. Dixon, J.J. Martin, J. Appl. Phys. 89, 5276 (2001)

5. J.S. McCullough, A.L. Harmon Bauer, C.A. Hunt, J.J. Martin, J. Appl. Phys. 90, 6017 (2001)

6. A.W. Wood, C.A. Hunt, J.J. Martin, J. Appl. Phys. 101, 063517 (2007)

7. A. Harmi, M. Secu, V. Topa, B. Briat, Opt. Mater. 4, 197 (1995)

8. B. Briat, T.V. Panchenko, H. Bou Rjeily, A. Hamri, J. Opt. Soc. Am. B 15, 2147 (1998)

9. F. Ramaz, L. Rakitina, M. Gospodinov, B. Briat, Opt. Mater. 27, 1547 (2005)

10. T.V. Pavchenko, Phys. Solid State 40, 415 (1998)

11. O. Kobozev, S. Shandarov, A. Kamshilin, V. Prokofiev, J. Opt. A, Pure Appl. Opt. 1, 442 (1999)

12. V. Marinova, S.H. Lin, V. Sainov, M. Gospodinov, K.Y. Hsu, J. Opt. A, Pure Appl. Opt. 5, S500 (2003)
13. S.M. Shandarov, A.E. Mandel', A.V. Kazarin, A.M. Plesovskikh, Yu.F. Kargin, V.V. Volkov, A.V. Egorysheva, Russ. Phys. J. 45, $763(2002)$

14. A.N. Dubrovin, A.E. Mandel', S.M. Shandarov, M.A. Zhnykina, Yu.M. Sukhoverkhov, Yu.F. Kargin, V.V. Volkov, A.V. Egorysheva, V.V. Shepelevich, Inorg. Mater. 40, 1280 (2004)

15. Yu.F. Kargin, A.V. Egorysheva, V.V. Volkov, M.N. Frolova, M.V. Borodin, S.M. Shandarov, V.M. Shandarov, D. Kip, J. Cryst. Growth 275, e2403 (2005)

16. A. Tolstik, A. Matusevich, M. Kisteneva, S. Shandarov, S. Itkin, A. Mandel, Yu. Kargin, Yu. Kylchin, R. Romashko, Quantum Electron. 37, 1027 (2007)

17. N.A. Vainos, S.L. Clapharm, R.W. Eason, Appl. Opt. 28, 4381 (1989)

18. A. Kamshilin, Opt. Commun. 93, 350 (1992)

19. A. Matusevich, A. Tolstik, M. Kisteneva, S. Shandarov, V. Matusevich, A. Kiessling, R. Kowarschik, Appl. Phys. B 92, 219 (2008)

20. R. Oberschmid, Phys. Stat. Sol. (a) 89, 263 (1985)

21. H.-J. Reyher, U. Hellwig, O. Thiemann, Phys. Rev. B 47, 5638 (1993)

22. H. Vogt, K. Buse, H. Hesse, E. Krätzig, Appl. Phys. 90, 3167 (2001)

23. A.L. Tolstik, N.G. Borzdov, A.Y. Matusevich, E.A. Melnikova, A.P. Sukhorukov, Nonlinear Optics Applications. Proc. of SPIE, vol. 5949 (2005), pp. 334-341 